\title{
North American Tropical Cyclone Landfall and SST: A Statistical Model Study
}

\author{
TIMOTHY HALL \\ NASA GISS, New York, New York \\ EMMI YONEKURA \\ Columbia University, New York, New York
}

(Manuscript received 18 October 2012, in final form 12 March 2013)

\begin{abstract}
A statistical-stochastic model of the complete life cycle of North Atlantic (NA) tropical cyclones (TCs) is used to examine the relationship between climate and landfall rates along the North American Atlantic and Gulf Coasts. The model draws on archived data of TCs throughout the North Atlantic to estimate landfall rates at high geographic resolution as a function of the ENSO state and one of two different measures of sea surface temperature (SST): 1) SST averaged over the NA subtropics and the hurricane season and 2) this SST relative to the seasonal global subtropical mean SST (termed relSST). Here, the authors focus on SST by holding ENSO to a neutral state. Jackknife uncertainty tests are employed to test the significance of SST and relSST landfall relationships. There are more TC and major hurricane landfalls overall in warm years than cold, using either SST or relSST, primarily due to a basinwide increase in the number of storms. The signal along the coast, however, is complex. Some regions have large and significant sensitivity (e.g., an approximate doubling of annual major hurricane landfall probability on Texas from -2 to +2 standard deviations in relSST), while other regions have no significant sensitivity (e.g., the U.S. mid-Atlantic and Northeast coasts). This geographic structure is due to both shifts in the regions of primary TC genesis and shifts in TC propagation.
\end{abstract}

\section{Introduction}

Intense tropical cyclones (TCs) are among the most devastating natural phenomena. Estimating the potential for economic damage is a topic of high public interest and cuts across meteorology, climatology, and economics (e.g., Mendelsohn et al. 2012; Peduzzi et al. 2012). Landfall risk assessments are used by the insurance industry for setting rates and by governments for establishing building regulations and planning emergency procedures. Given the large coastal populations in harm's way, estimating the short- and longterm evolution of TC hazard is crucial.

There has been much interest in ascertaining and understanding trends in North Atlantic TC frequency and intensity and whether any such trends are related to anthropogenic climate change and/or natural climate cycles. North Atlantic (NA) sea surface temperature (SST) has risen in recent decades, driven in large part by

Corresponding author address: Timothy Hall, NASA GISS, 2880 Broadway, New York, NY 10025.

E-mail: timothy.m.hall@nasa.gov greenhouse gas forcing (Santer et al. 2006). Some statistical studies have suggested a link between SST and NA TC activity (Emanuel 2005; Elsner et al. 2008), while other studies point to the spatial structure of SST as being more important than absolute SST (Vecchi and Soden 2007; Vecchi et al. 2008; Villarini et al. 2010; Ramsay and Sobel 2011; Villarini et al. 2012). Directnumerical and downscaled-numerical transient climate simulations of the twenty-first century using the Intergovernmental Panel on Climate Change (IPCC) models produce widely varying TC results (Emanuel et al. 2008; Camargo 2013; Villarini and Vecchi 2012). The consensus among the climate models that are best at representing TCs is for reduced TC frequency by 2100 but an increased fraction of TCs in the highest intensity categories (cat; Bender et al. 2010; Villarini and Vecchi 2013).

Whatever are the long-term trends in large-scale measures of TC activity, and however they are driven, it is ultimately necessary for risk assessment to estimate landfall rates at local geographic scales. This task is beyond the capability of global climate models. The most straightforward way to analyze landfall risk and its climate dependence is to use historical landfall events.

DOI: 10.1175/JCLI-D-12-00756.1 
This approach is sound if there are a sufficient number of events, such as are found over large sections of coast and over many years. However, if one aims to study geographic landfall rates at high geographic resolution and in addition use subsets of data years based on climate state, then sampling error becomes a major issue. This can be alleviated by exploiting data from outside the immediate region of interest, for example by supplementing the local landfall wind data with overocean data (e.g., Emanuel and Jagger 2010). Another alternative is basinwide statistical models of the entire life cycle of TCs to generate sets of synthetic TCs much larger than the historical set and compute landfall probabilities from the synthetic sets. Various such models have been developed by private and public sector researchers (James and Mason 2005; Vickery et al. 2006; Emanuel et al. 2006; Rumpf et al. 2007).

An advantage of a basinwide model is that it utilizes historical track information over the full basin: orders of magnitude more data than just at landfall. In effect, misses, as well as hits, inform the landfall analysis. A disadvantage is that while reliable landfall data go back into the nineteenth century, full-basin data are less reliable prior to the mid-twentieth century. In addition, the added complexity of a full-basin model compared to a landfall model increases the possibility of model bias. Still, for coastal regions of historically few or no landfalls, a basinwide model is beneficial, as seen in the analysis of Hall and Jewson (2008), who show in out-ofsample tests that the increased precision outweighs any loss of accuracy. A second advantage to simulating the TC full life cycle is that landfall changes can be decomposed into changes in various TC components, such as genesis, propagation, and intensity. Here, the model initially described by Hall and Jewson (2007) is extended and used to explore the relationships between regional landfall rates and SST.

Section 2 reviews the data on which the model is built, and section 3 describes the statistical-stochastic model, focusing on components that have not been documented elsewhere. The model is evaluated in section 4 , and the landfall-SST relationships explored in section 5 .

\section{Data sources}

We use the Atlantic hurricane database (HURDAT) best-track data from 1950 to 2008 (Javinen et al. 1984) to build the statistical model. These data include position, central pressures, and maximum sustained wind speeds $\left(V_{\max }\right)$ every $6 \mathrm{~h}$ along a TC's trajectory. We restrict attention to named TCs. The date 1950 represents roughly the start of the era of routine aircraft reconnaissance. Moreover, Vecchi and Knudson (2011) have estimated that prior to 1950 the number of TCs missed by undersampling increases substantially. Importantly, all HURDAT storms that survive less than 2 days are excluded from the analysis. There is evidence that changes in the frequency of such short-lived storms are spurious, due to underreporting earlier in the record (Villarini et al. 2011), and we wish to avoid such ambiguity in the results. As part of the track propagation component of the model we use a composite annual cycle of 500-mb zonal wind, which has been constructed from 1950 to 2008 6-hourly National Centers for Environmental Prediction (NCEP) wind data.

We employ SST and the Southern Oscillation index (SOI) as independent predictor variables, both of which are known to influence TC activity. In this study, we focus on SST and hold SOI to a neutral value for all experiments. Other factors have been shown to influence NA TC activity as well [e.g., the solar cycle and the North Atlantic Oscillation (NAO); Elsner et al. 2006; Elsner and Jagger 2008].

There has been considerable debate as to how SST should be viewed in relation to NA TC activity (Vecchi et al. 2008). The question is of enormous importance for long-range projections of TC activity, given the robust projections of increased SST over the next several decades. Recent work has emphasized the importance of spatial structure in SST, rather than SST directly, as being the best indicator for TC activity based on physical arguments and numerical simulations (Vecchi et al. 2008). In particular, relative SST (relSST), the NA SST compared to the global tropical mean SST, has been shown to be at least as good a statistical predictor of NA TC counts as SST directly (Villarini et al. 2010; Vecchi and Knudson 2011; Villarini et al. 2012). There is no reason to expect a robust signal in relSST due to the radiative forcing of well-mixed greenhouse gases, though aerosol forcing is known to have affected the NA disproportionately in the twentieth century (Villarini and Vecchi 2012). One physical argument for relSST is that it is a proxy for potential intensity (PI; Vecchi and Soden 2007): a measure of the upper limit of TC intensity (Holland 1997; Emanuel 2000) and a factor in TC genesis (Camargo et al. 2007).

For an analysis of recent trends and near-term projections, however, the choice between SST and relSST is less important than it is for long-range projections. In the near term, natural variability dominates anthropogenic trends, NA SST and relSST are well correlated, and either one may be acting as a convenient proxy variable for modes of climate variability that affect TCs via several mechanisms. In this study, we perform separate analyses using as independent variables SST directly and relSST. Gridded SST data are obtained from 


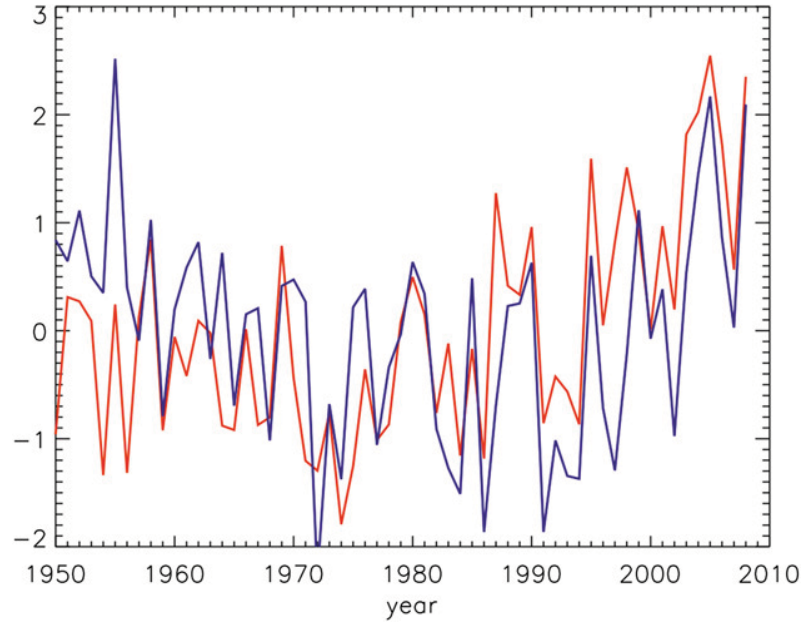

FIG. 1. Time series of NA MDR JASO mean SST (red) and NA MDR JASO mean relative SST (blue).

the Met Office Hadley Centre (Rayner et al. 2003) and are averaged over the North Atlantic main development region (MDR) and the hurricane season July-October (JASO) to obtain annual values. For relSST, JASO $20^{\circ} \mathrm{S}$ to $20^{\circ} \mathrm{N}$ global averages are subtracted from MDR-JASO SST. As will be shown from the perspective of modeling current climate TC landfall rates statistically, use of SST and relSST yield similar results because SST and relSST are well correlated (Fig. 1). At several key points in the paper we show results for both SST and relSST.

\section{Methodology}

The goal of this study is to estimate the sensitivity of local landfall rates along the North American coast to NA SST and relSST. This is difficult using solely TC data at landfall because there are too few such data on a local basis. Instead we make use of an SST-sensitive statisticalstochastic model for TCs over the full NA (i.e., a statistical model of TCs from birth to death that draws on basinwide data). In this way, much more data are exploited than just landfall. In effect, information from data over the full basin is projected onto the coast. Once constructed, the model is run to generate "event sets" of synthetic TCs that are much larger than the historical set, and these synthetic TCs are used to compute landfall rates. Large event sets and corresponding landfall rates are generated for a range of fixed SST and relSST. The model components also depend on SOI; in this study we focus on the SST effects and hold SOI at a constant neutral value (zero anomaly value) for all experiments.

The four components of the TC model are 1) genesis, 2) propagation, 3) lysis, and 4) intensity. The genesis, propagation, and lysis components are similar to those described by Hall and Jewson (2007), with updates described by Yonekura and Hall (2011, 2013, manuscript submitted to J. Appl. Meteor. Climatol.). The intensity component is new. The basic scheme for generating a synthetic TC is to first simulate a genesis event. Then, from the genesis location the TC is propagated in 6-hourly increments. At each 6-h position, the model determines whether or the not the storm suffers lysis (terminates), continuing until lysis occurs. For the TC intensity, a time series of the maximum sustained wind speed $\left(V_{\max }\right)$ is placed on the track. The model components are discussed individually below, with the most detail reserved for the new intensity scheme.

\section{a. Genesis}

The genesis component is identical to that described in Yonekura and Hall (2011) for the northwestern Pacific, which in turn is a generalization of the Hall and Jewson (2007) steady-state kernel density genesis to the case of climate-dependent variation. We perform local Poisson regression of the annual number of TC genesis events on SST or relSST and SOI. In a data circle of radius $L$ centered on location $r$, an annual time series of genesis counts is Poisson regressed on SST and SOI, and the resulting Poisson rate is divided by the area of the circle to obtain
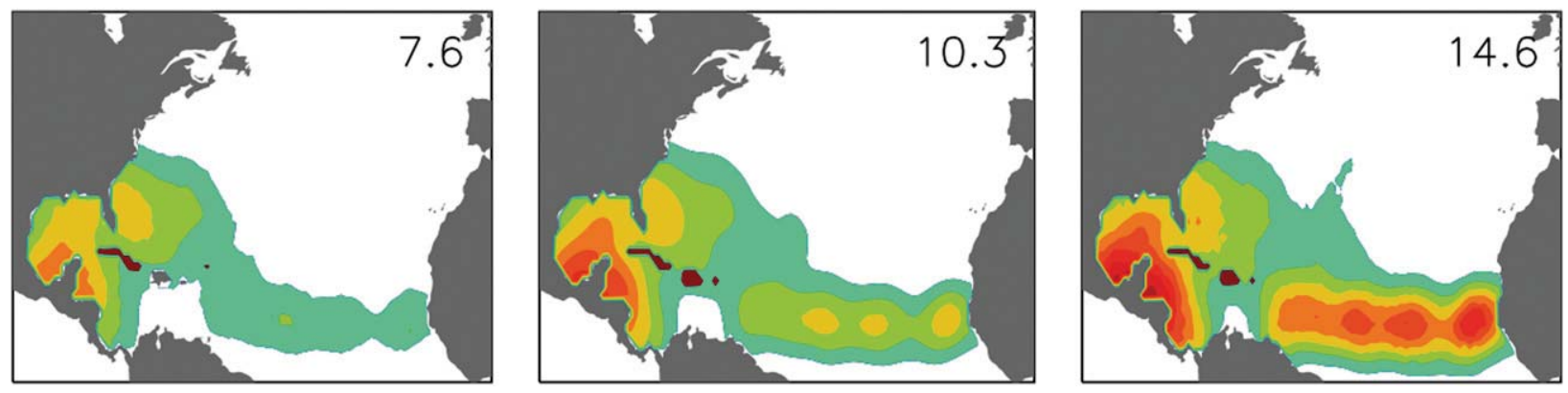

FIG. 2. Genesis rates and mean annual count (inset numbers) for SST $=($ left $)-2$, (middle) 0 , and (right) +2 in units of standard deviations in JASO MDR SST over 1950-2009. Contour intervals are 0.003, 0.006, 0.009, 0.012, 0.015, and 0.018 annual counts per 1-degree longitude-latitude box from light blue to dark red. White regions correspond to values below 0.003 . 

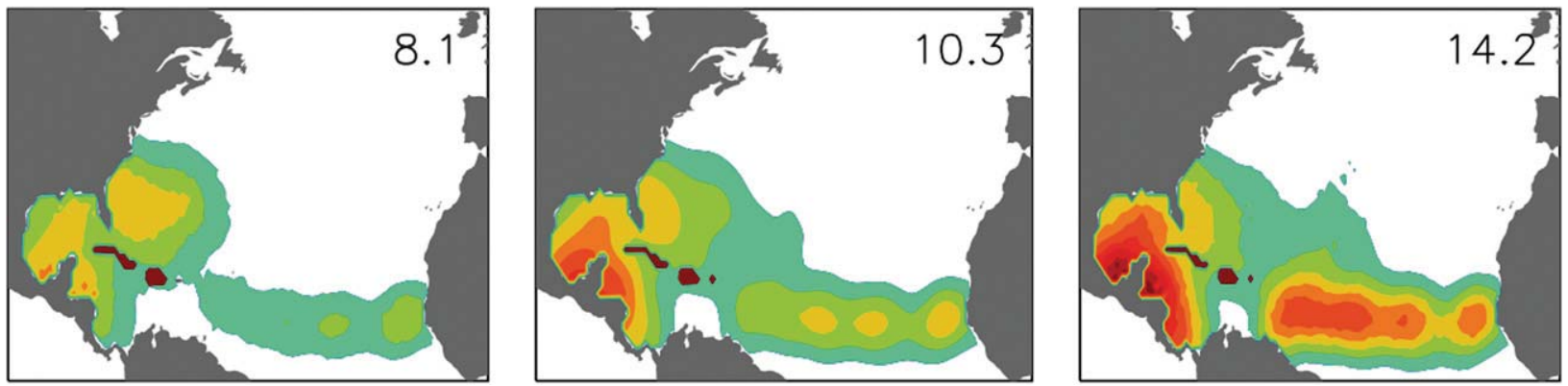

FIG. 3. As in Fig. 2, but for those dependent on relative SST.

a rate per area. We then multiply by the area of a $1^{\circ}$ box about $r$ to obtain a grid of Poisson rate coefficients spanning the domain of interest $\left(5^{\circ}-55^{\circ} \mathrm{N}, 100^{\circ} \mathrm{W}-0^{\circ}\right)$. During simulations the appropriate value of SST and SOI are multiplied by the local coefficients to obtain local rates. The Poisson distribution defined by this rate is then sampled randomly to obtain a count of genesis events for the grid box for the year. The counts are almost always 0 or 1 , given the small region, though in principle higher counts can occur.

The radius $L$ of the local data circle in which historical counts are included for regression is determined by out-ofsample likelihood maximization and is equal to $700 \mathrm{~km}$ for
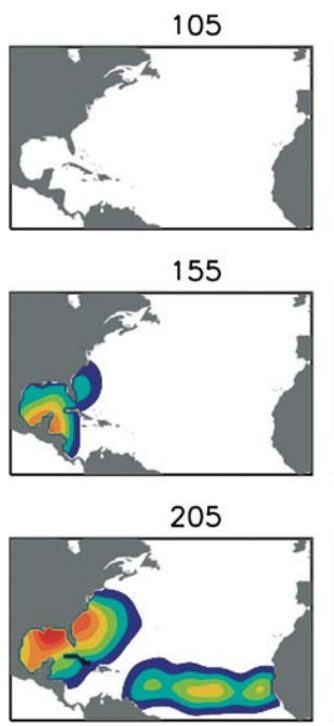

255

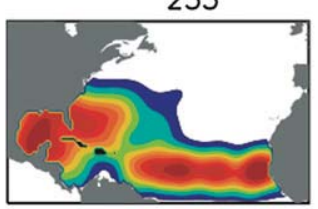

305
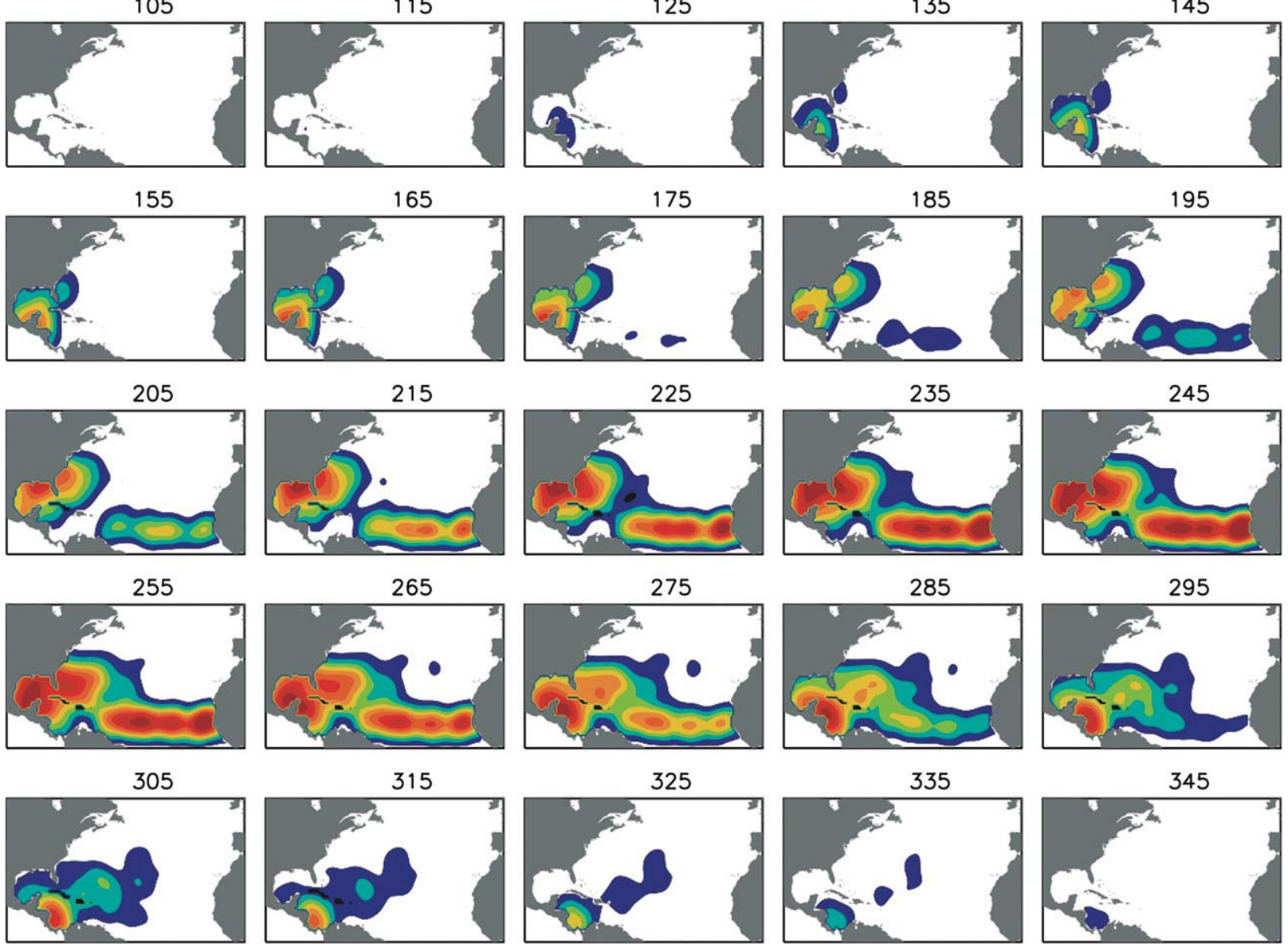

195
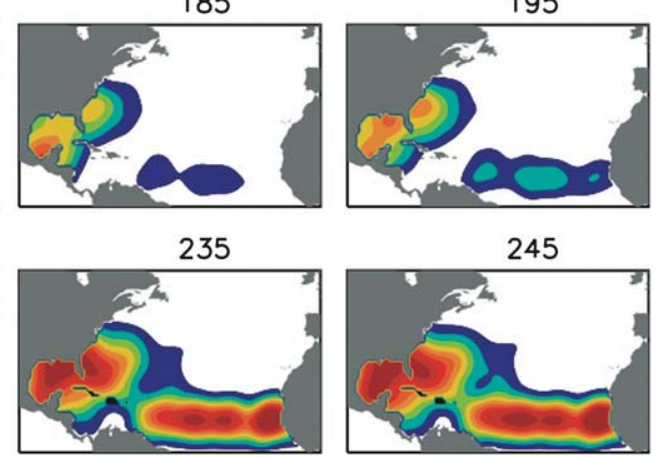

FIG. 4. The annual cycle of formation rates from an optimized space-date kernel density. Spatial fields are shown for 10-day windows centered on the day of year shown above each panel. For plotting, the kernel density is scaled to have the unit maximum, and the contour intervals are $0.1,0.2, \ldots, 0.9$, blue to red. White regions correspond to values below 0.1 . 
(a)

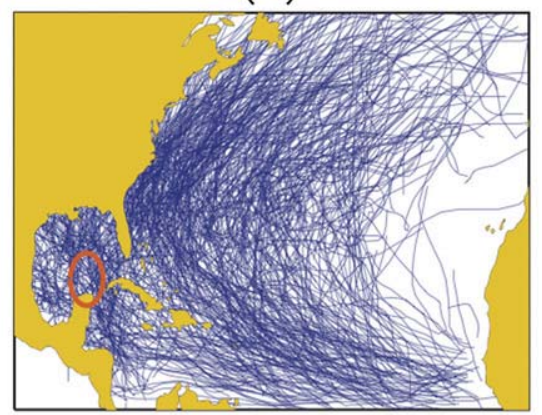

(c)

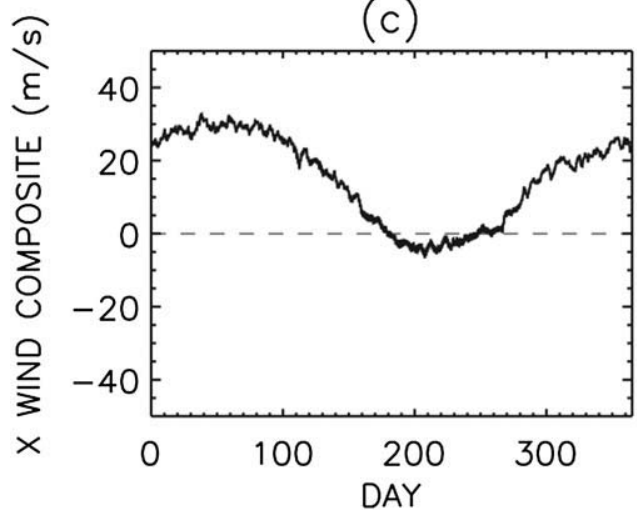

(b)

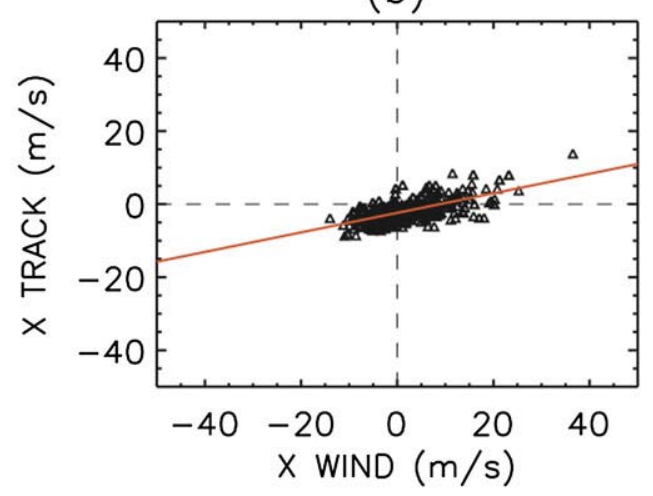

(d)

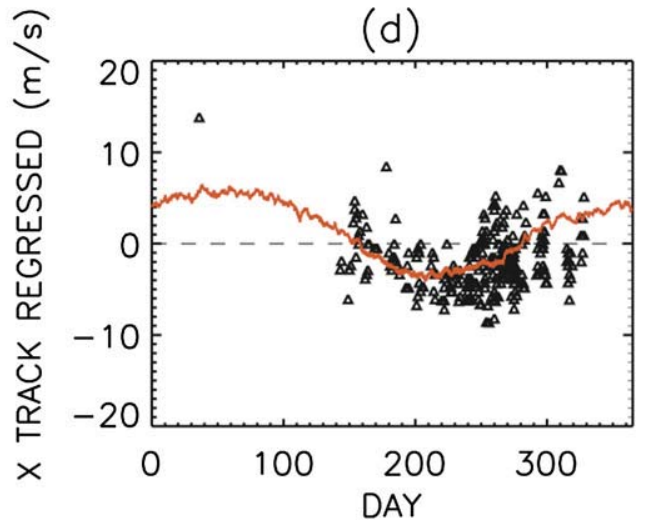

FIG. 5. Illustration of the dependence of track propagation on the annual cycle of 500-mb zonal winds. (a) Historical tracks (blue) and a sample local regression region (red) in the Gulf. (b) Scatterplot of 6-houly zonal propagation speed vs the concurrent 500-mb annual cycle wind speed and the regression line (red). (c) Time series of the annual cycle zonal wind over the region. (d) Time series of the track speed predicted by the regression (red) and the actual track speeds (symbols).

the 1950-2008 period. This course scale blurs the sharp latitudinal gradient of the formation rate at the southern flank of the MDR. The Poisson coefficient fields are "refocused" by forcing the local rates to conform to a steadystate kernel density field calculated from the 1950-2008 data, which has an optimized bandwidth of $210 \mathrm{~km}$. In effect, though the climate dependence of the genesis is less well resolved, the mean rates at least are constrained by the better-resolved historical averages. Genesis is prohibited over land on physical grounds. Overland power that results from the isotropic-averaging kernel is truncated and redistributed nearby over ocean.

Figure 2 shows the genesis rate distribution for SST standardized anomalies of $-2,0$, and +2 . There is an approximate doubling of the basinwide mean formation rate from SST $=-2$ to $+2\left(7.6\right.$ to $\left.14.6 \mathrm{TCs} \mathrm{yr}^{-1}\right)$. The rate does not increase uniformly. Rather, there is a greater increase in the MDR, resulting in an effective shift southeastward of the center of genesis activity. This genesis site shift has also been identified by Kossin and Vimont (2007) and Wang et al. (2011), who argue that it is a consequence of an eastward shift of the Atlantic warm pool [and by extension and increased MDR JulySeptember (JAS) SST], as well as associated reductions in wind shear, possibly connected to the Atlantic meridional mode (AMM; Kossin and Vimont 2007). Figure 3 shows the same genesis maps but now with relSST as the independent variable. The resulting sensitivity is similar to SST, with a $10 \%$ reduced overall formation rate change (8.1 to $14.2 \mathrm{TCs} \mathrm{yr}^{-1}$ ). (There is a small component of the relSST series that is linearly related to SOI: SOI explains about $20 \%$ of the variance in relSST. When we use a "corrected" relSST signal-relSST with this linear component removed - the results are not significantly different than Fig. 3.)

We also include seasonality in the genesis model (i.e., the date during the year the event occurred). This is accomplished with a 3D (longitude, latitude, and date) kernel density. The date dimension of the kernel density is randomly sampled at each location to provide a date for the genesis event. Figure 4 shows cuts of the spatial density at 25 10-day windows spanning the hurricane season. 

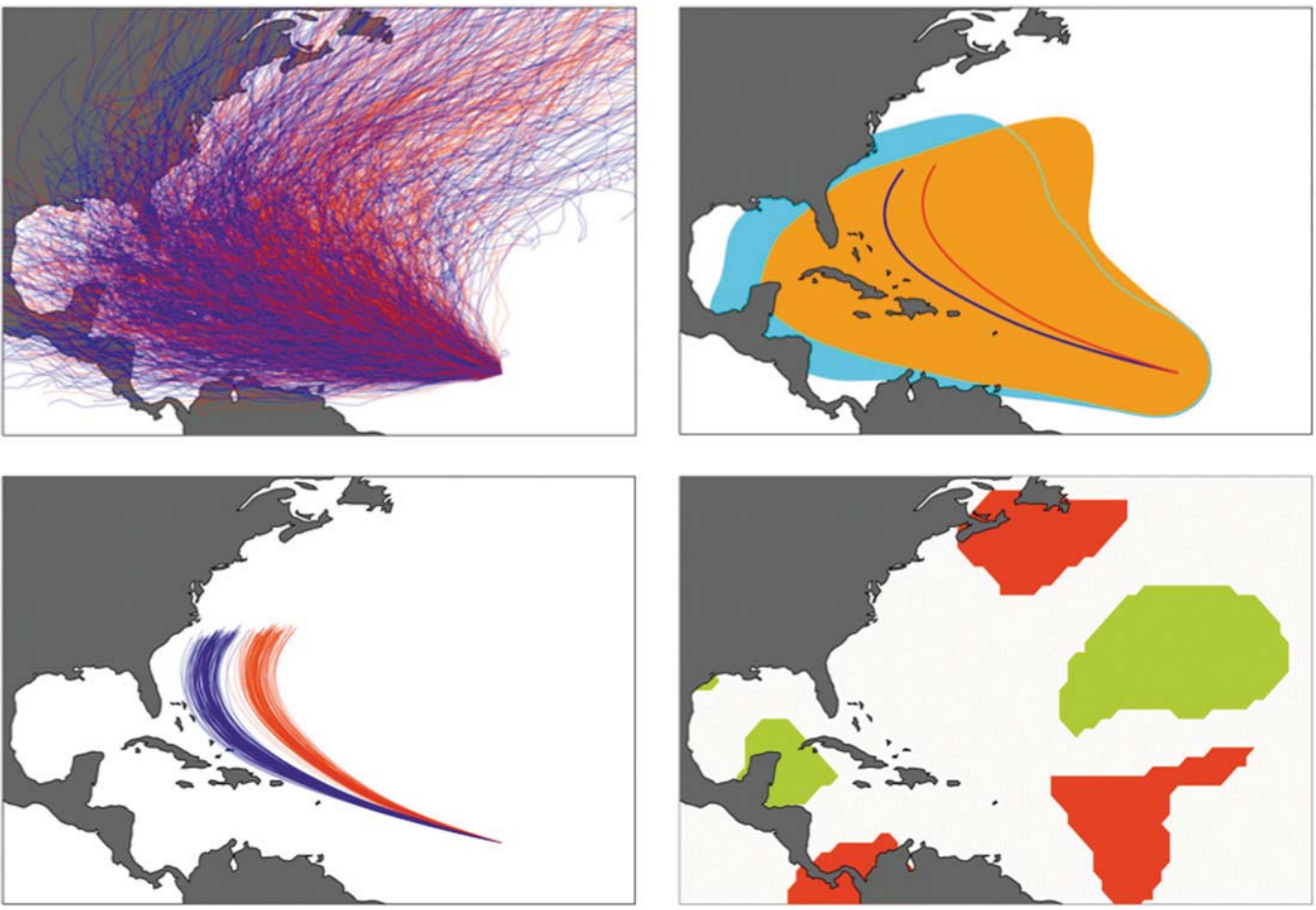

FIG. 6. (top left) The 1000 synthetic tracks for +2 (red) and -2 (blue) SST emanating from a single point. (top right) The SST +2 and -2 mean tracks (red and blue curves, respectively and the track-point density above a threshold (orange and light blue, respectively) about the +2 and -2 means. (bottom left) The +2 and -2 mean tracks (red and blue, respectively) for a 100-member jackknife uncertainty set. The means are significantly different to the extent the distributions of means across the jackknife set are distinct, which they clearly are. (bottom right) Red (green) indicates regions of significant westerly (easterly) shift in mean track vectors with increased SST. In other regions, the propagation-SST relationship is not significant. Significance is determined by the same jackknife procedure.

\section{b. Propagation}

TC propagation is identical to that described by Yonekura and Hall (2011) for the NWP, which is a generalization of the Hall and Jewson (2007) track model. Given a TC at location $r$, the two vector components of the 6-hourly increment $d r$ to the new position $r+d r$ are determined by linear multiple regression of nearby (to be determined objectively) HURDAT increments on SST (or relSST), SOI, and 500-mb zonal wind speed from an NCEP-based composite annual cycle. Regression on this composite, illustrated in Fig. 5, provides seasonal dependence of the propagation. The residuals are standardized, assuming a generalized anisotropic correlated normal process, as illustrated in Fig. 3 of Yonekura and Hall (2011). The standardized residuals are modeled by lag-one autoregression [AR(1)]. HURDAT contributions to the regression at $r$ are weighted inversely with distance from $r$ using a Gaussian kernel whose length scale is optimized by an out-of-sample likelihood maximization. The $500-\mathrm{mb}$ wind-independent variable is used because midlevel winds are known to steer TCs.
The annual cycle provides a mechanism to reproduce seasonality in tracks.

There is a statistically significant relationship between SST and track propagation in parts of the domain, as illustrated in Fig. 6. Tracks have large stochastic components, and launching many tracks from a fixed point in the middle MDR leads to a wide and overlapping spread of tracks, from which it is difficult to discern any impact of SST. When track-point density is contoured on the domain, there appears to be a slight shift away from the North American coast from cold to warm years. This shift is seen more clearly when just the mean tracks are plotted (i.e., the stochastic component is set to zero). However, alone it is not clear if this difference is significant. To test the significance we perform a generalized jackknife test. The regression of HURDAT tracks on the independent variables is performed 100 times, each time dropping a random $14(\sim 20 \%)$ of the 69 data years. (The fraction $20 \%$ is an arbitrary compromise between two competing factors: the fraction should be large so that there are many possible permutations for the subset years from the full set, and the fraction should 


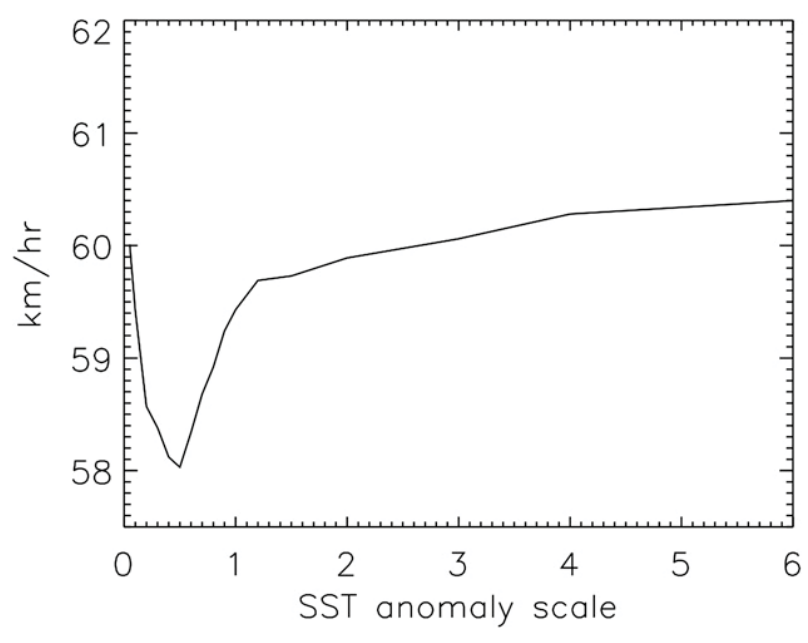

FIG. 7. Root-mean-square forecast error in maximum $V_{\max }$ along tracks as function of SST anomaly scale. A shallow minimum occurs at $S=0.5$.

be small so that there are many data within the subset to perform regressions.) For each 55-yr subset, the mean tracks are simulated from the selected point for SST $=+2$ and -2 . The result is shown in Fig. 6 (lower left). Clearly, the jackknife hot and cold sets are distinct, and the shift in mean track with SST is significant, at least from this selected location. Finally, the jackknife procedure is repeated for each location on a $1^{\circ}$ by $1^{\circ}$ grid, using single-step $(6 \mathrm{~h})$ mean tracks. The lower right panel of Fig. 6 shows regions where the SST dependence is significant: red indicating an eastward shift in propagation and green indicating a westward shift in propagation.

Wang et al. (2011) find a similar effect of SST on track propagation using dynamical simulations. They argue that a large Atlantic warm pool (and by extension a warmer MDR JAS mean SST) leads to a stationary wave pattern that weakens the NA subtropical high. A weakened subtropical high in turn induces an eastwardsteering flow anomaly in the western Atlantic off the North American coast. Similarly, Colbert and Soden (2012) note an increase in recurving versus straightmoving TCs from the MDR with a weakened subtropical high, a change in propagation that they also associate with the AMM. The weaker relationship we find between track and relSST (not shown) may simply be a signature of the track-SST relationship, via the SST-relSST correlation.

c. Lysis

At each 6-h position $r$, along a simulated track, the TC has a probability of terminating. The probability $P$ is derived from analysis of the historical TCs that have terminated near $r$, as described in Hall and Jewson (2007):

$$
P=\frac{\sum_{\text {term }} e^{-\left(r-r_{i}\right)^{2} / L}}{\sum_{\text {all }} e^{-\left(r-r_{i}\right)^{2} / L}},
$$

where the sum in the denominator is taken over all TC points, and the sum in the numerator is taken only over terminal TC points. The length scale $L$ determines how locally the sums should be weighted and is determined by out-of-sample log-likelihood maximization. The meteorological mechanisms for TC dissipation over water and land are very different. Therefore, land and ocean lysis probabilities are strictly separated using a $0.1^{\circ}$ land-ocean mask.

Note that in formulation (1) the TC's intensity does not appear. However, the time series of $V_{\max }$ that gets placed on simulated tracks (see below) is constrained by the lysis location such that intensity at lysis is realistic.

\section{d. Intensity}

The model component for time series of the maximum sustained wind speed $\left(V_{\max }\right)$ is different in character than the other components. Instead of regression, we employ a scheme to resample the HURDAT $V_{\max }$ time series. First, a track is simulated using the genesis, propagation, and lysis schemes described above. Then, a $V_{\max }$ series is selected among the HURDAT series by a weighted random draw, and this series is placed on the simulated track, with rescaling in time to match the duration of the simulated track. Finally, a random perturbation to the maximum $V_{\max }$ along the series is made, and the entire series is rescaled to match the new perturbed maximum $V_{\max }$ but holding the first and last points unchanged.

The random draw of a HURDAT TC's $V_{\text {max }}$ series is weighted toward HURDAT TCs whose tracks are similar to the simulated track on which the $V_{\max }$ series is to be placed. Similarity is defined by several criteria: 1 ) the proximity of the simulated and historical genesis sites, 2) the proximity of the simulated and historical lysis sites, and 3) the proximity of the amount of time that simulated and historical TCs spend in different latitude bands. If a track makes one or more landfalls, then it is broken into overocean and overland segments, and separate weighted $V_{\max }$ random draws are made for each segment. In such landfalling cases additional sampling weights are used: 4 ) the proximity of the simulated and historical landfall locations and 5) the proximity of the last overocean $V_{\max }$ value to the first overland $V_{\max }$ value. If the simulated $\mathrm{TC}$ reemerges over the ocean, 
(a)

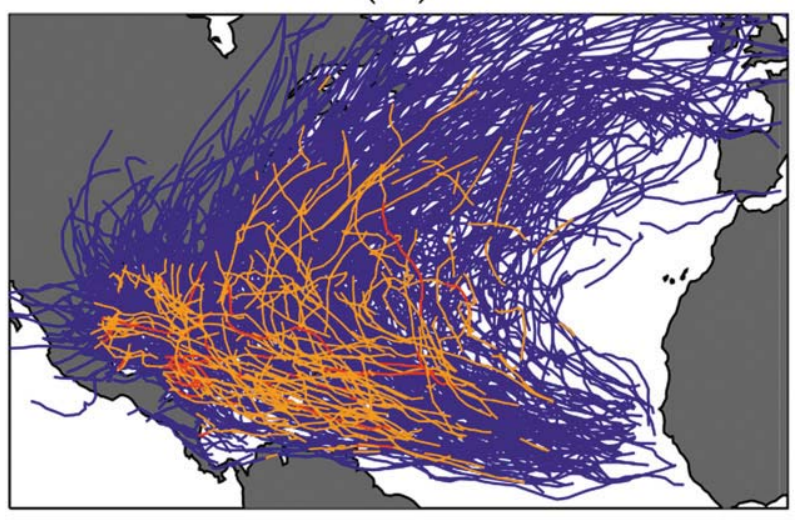

(c)

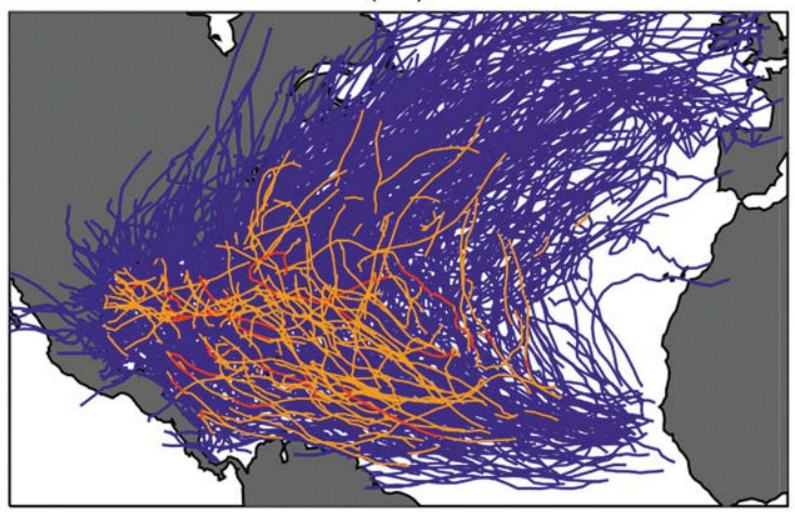

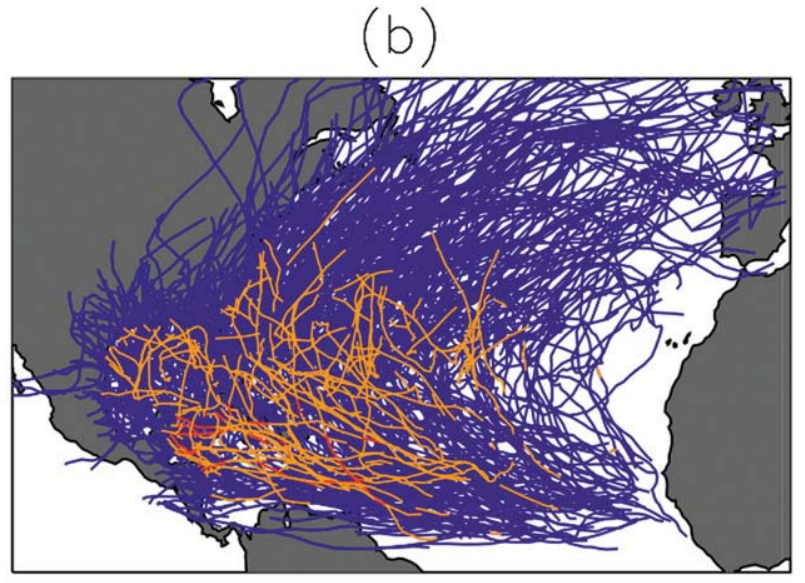

(d)

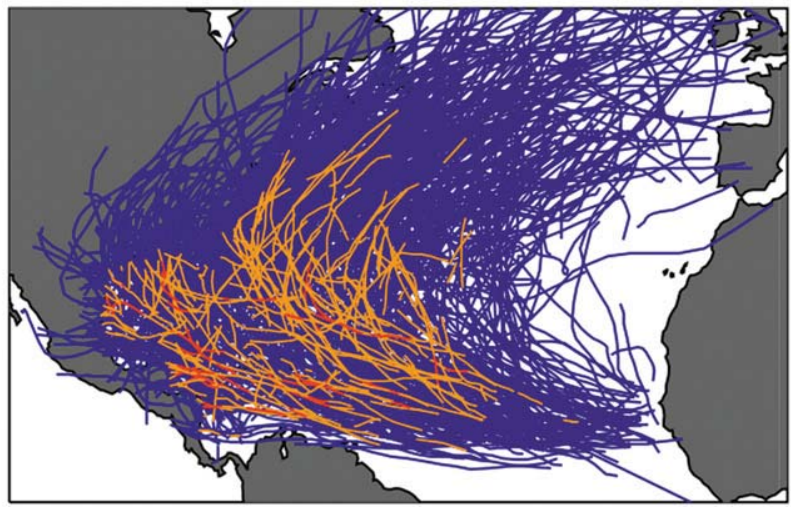

FIG. 8. (a)-(c) Three randomly selected examples from 1950-2010 simulations, (d) along with the HURDAT tracks. Blue corresponds to cat- $0-2$, yellow cat- $3-4$, and red cat -5 .

then the new $V_{\max }$ series are selected for the reemergent $\mathrm{TC}$, again using proximity weights at the point of reemergence. The transition from one $V_{\max }$ series to another ocean to land and land to ocean is made smooth by rescaling the new segment to match the prior segment at the transition point.

We want to allow for direct sensitivity to the independent climate variables in the $V_{\max }$ selection. (There is already indirect sensitivity. The genesis sites change with SST and SOI, and different $V_{\max }$ series will be preferentially selected.) Therefore, a final selection weight is added: the proximity of the SST (or relSST) anomaly from the year of the candidate $V_{\max }$ series to the SST (or relSST) anomaly of the current simulation year. That is, in the random draw a candidate $V_{\max }$ series $i$ gets weighted by $\exp \left[-\left(\mathrm{SST}_{\mathrm{sim}}-\mathrm{SST}_{i}\right) / L\right]$, where $\mathrm{SST}_{\text {sim }}$ is the SST anomaly of the simulation year, $\mathrm{SST}_{i}$ is the SST anomaly of the year of the candidate $V_{\max }$ series, and $L$ is a scale to be determined. (The quantity $L$ is dimensionless, as the SSTs are expressed as standardized anomalies.)

Does the addition of such an SST weight lead to improvement in model forecast or overconstrain the model? To answer this, we perform an out-of-sample year optimization of the scale $L$ used to define SST proximity. On the one hand, if it turns out that the optimal $L$ is infinite, then $V_{\max }$ series from all years, regardless of SST (or relSST), should be used equally, and preferential weighting by SST is overconstraining. On the other hand, if the optimal $L$ is finite, then the SST (or relSST) state is important enough to warrant selecting from a $V_{\max }$ dataset effectively smaller than the full set.

The procedure is as follows:

1) Pick an SST anomaly scale $L$.

2) Pick an out-of-sample year in 1950-2008 and loop through the historical tracks of that year.

3) For each track, select a $V_{\max }$ series from the remaining years. 


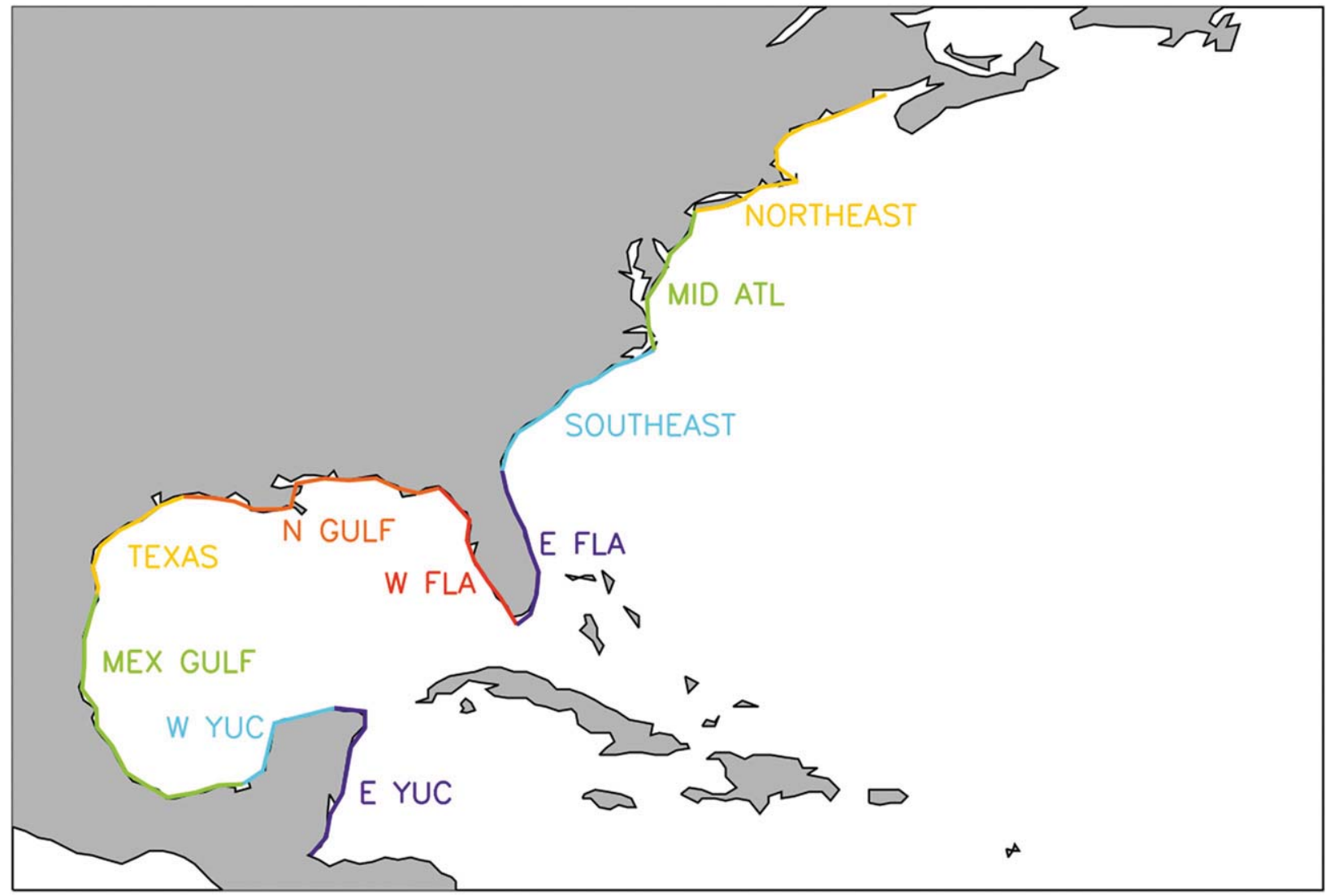

FIG. 9. Map illustrating 87 coast segments along the Caribbean, Atlantic, and Gulf Coasts. The segments are approximately $100 \mathrm{~km}$ in length. They are divided into 10 regional categories, through color codes and labels.

4) Compute the difference of the maximum $V_{\max }$ of the selected series from the maximum $V_{\max }$ of the historical series.

5) Accumulate the squared differences and record the root-mean-square average as the out-of-sample forecast error for the anomaly scale.

6) Minimize the error in $L$.

This type of optimizing is similar to the optimization performed in the selection of scales defining the locality of regression in the track, genesis, and lysis models (Hall and Jewson 2007). There is a balance between two competing factors: 1 ) a large $L$ includes as much data as possible, thereby reducing sampling error; and 2) a small $L$ resolves underlying meteorological dependence of TC intensity on SST. Figure 7 shows the error as a function of the SST anomaly scale. There is a shallow minimum at $L=0.5$, indicating that $V_{\max }$ has enough sensitivity to SST to warrant the inclusion of SST as a weight, despite the increased sampling error.

In this resampling scheme for $V_{\max }$, the maximum intensity possible for a simulated storm is the maximum intensity recorded in the HURDAT database. This is a limitation and is not in keeping with the stochastic nature of the other model components, which are not bounded by historical values. To remedy this we add random perturbations to the maximum $V_{\max }$ values achieved along TCs [lifetime maximum intensity (LMI)], rescaling each storm's $V_{\max }$ series accordingly, such that the first and final $V_{\max }$ values are unchanged. This rescaling, however, needs to be done carefully, so that the distributional character of the resulting set of LMI is unchanged. We do not want the resulting set of simulated LMI to have a frequency distribution different in character than the historical set of LMI.

First, we perform a simulation and store the set of as yet unperturbed LMI. This unperturbed simulation set has a frequency distribution of LMI that matches the historical distribution, assuming the track simulations are realistic, so that an unbiased sample of the $V_{\text {max }}$ series is selected. Following Casson and Coles (2000), we model the LMI with a generalized extreme value (GEV) distribution fit to the historical LMI. GEV is the appropriate distribution for the maximum in a series of 

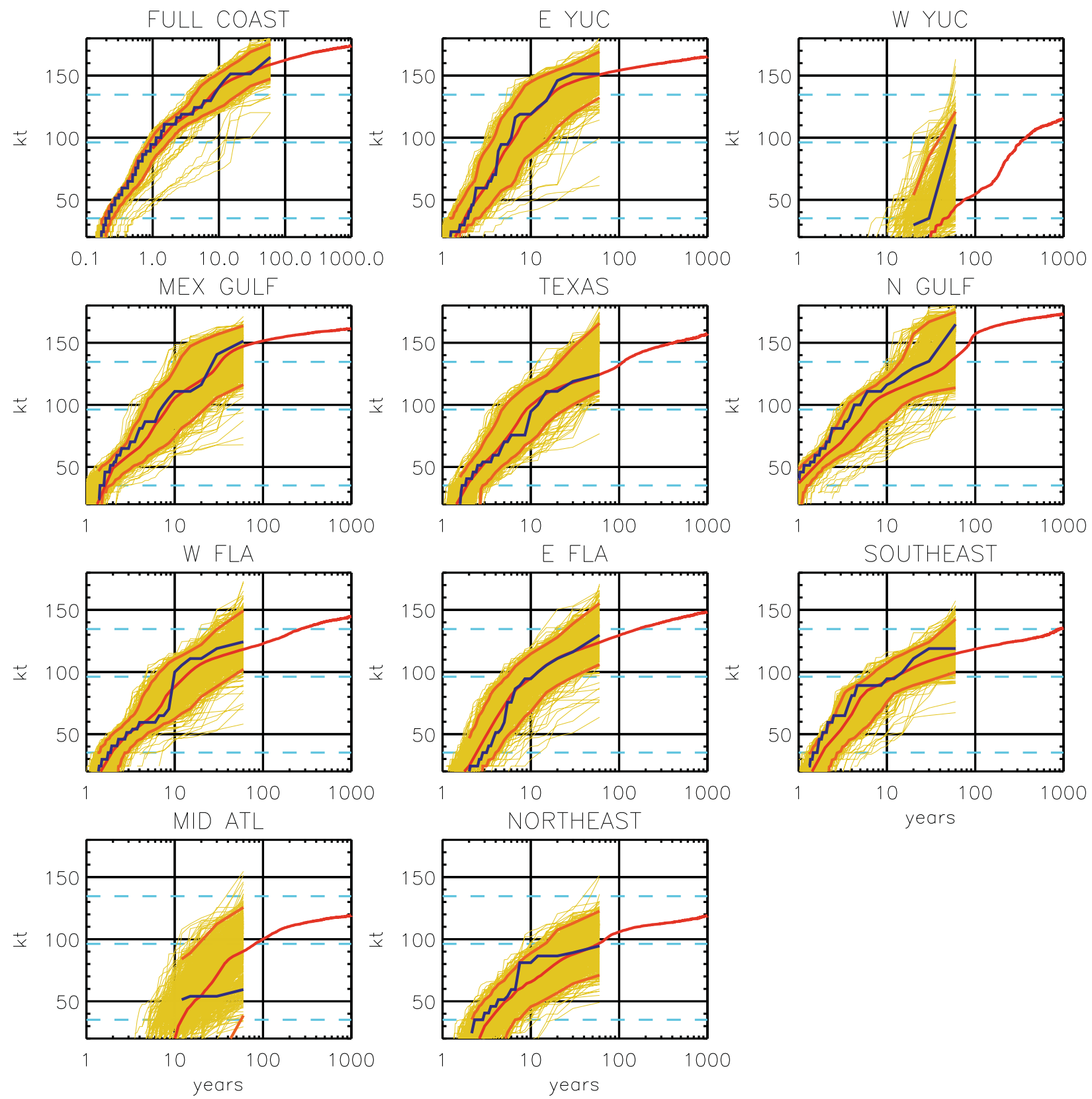

FIG. 10. Landfall return-period curves for the full coast and each of the 10 regions, as labeled. Yellow curves are shown for each of the 5000 simulations of the 59-yr (1950-2008) period. The orange curves indicate the lower and upper bounds of the inner $95 \%$ across the 5000 simulations. The red curve is obtained by combining the 5000 simulations in series (i.e., a 295000 -yr simulation). The blue curve is obtained directly from HURDAT landfalls. Model landfall is unbiased to the extent that the blue curve falls inside the orange curves.

random values. The GEV distribution is then converted to normal by matching cumulative distribution functions (CDFs). (That is, each maximum $V_{\max }$ value in the GEV has a CDF value $Y$. The quantity $X$ is found, such that the standard normal CDF at $X$ equals $Y$.) The elements of the normal set are perturbed with a normal random of zero mean and standard deviation $\sigma$, which is determined objectively by an out-of-sample minimization of the LMI forecast error. The perturbed normal set is then standardized. Finally, the standard normal perturbed set is converted back to GEV, again by $\mathrm{CDF}$ matching. This results in a set of $V_{\max }$ series on simulated tracks whose maximum $V_{\max }$ is not bounded by the highest observed $V_{\max }$ but whose frequency distribution has a form that matches that of the historical maximum $V_{\max }$. 


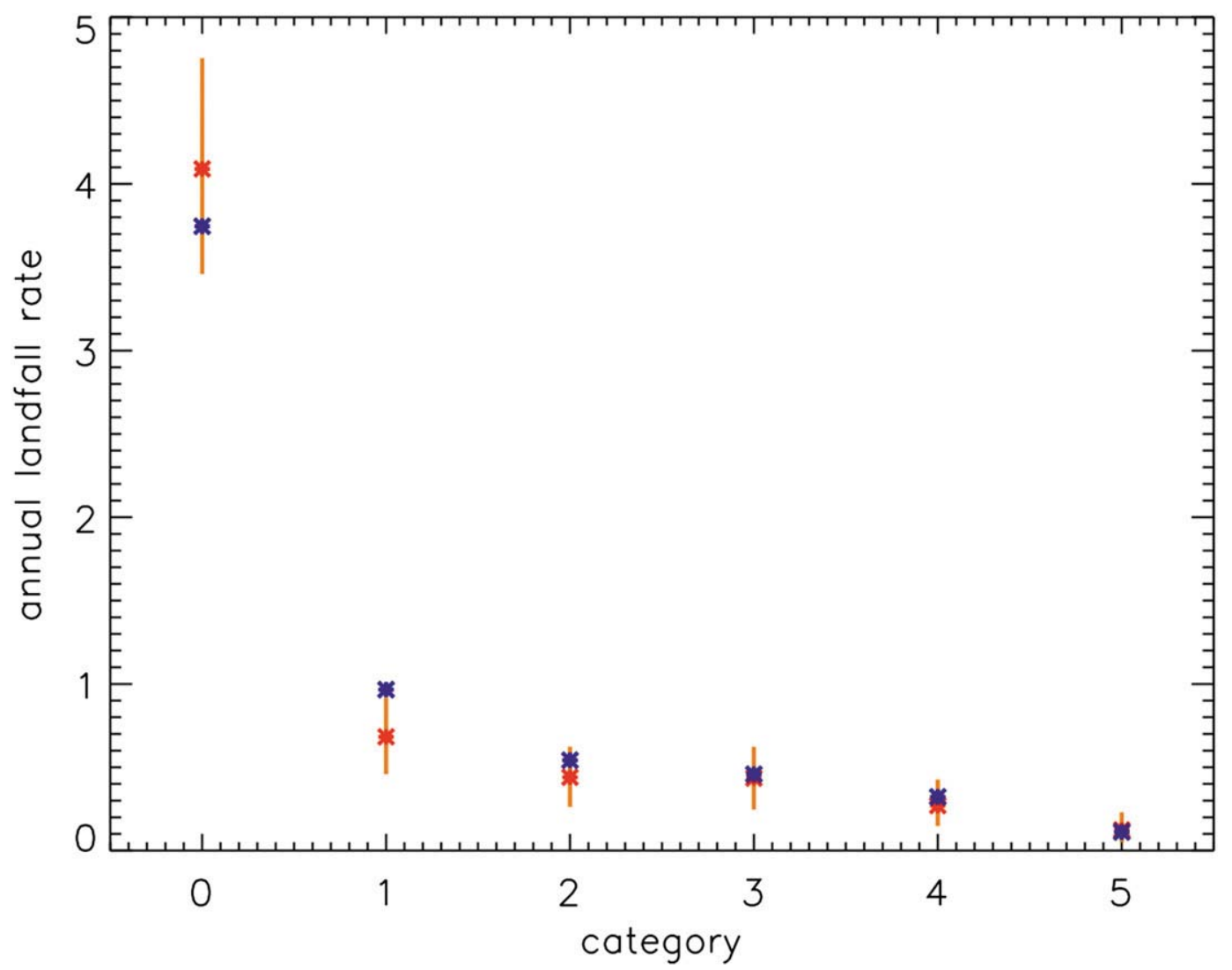

FIG. 11. Annual landfall rates (counts $\mathrm{yr}^{-1}$ ) over the full coast (Yucatan through U.S. Northeast) of Fig. 10 separately in each intensity category 0 to 5 . The red symbols are the mean of the 5000-member 1950-2008 simulation set. The orange bands show the $95 \%$ ranges about these means. The blue symbols are the rates directly computed from HURDAT.

\section{Evaluation}

Before using the TC model to estimate landfall rate sensitivity to SST and relSST, it is important to evaluate the model. The 1950-2010 period is simulated 5000 times and the results compared to the historical TCs. Tracks from three such simulations are shown in Fig. 8, along with the historical tracks. The model performs well according to a specified diagnostic to the extent that the historical diagnostic value appears as a typical member of the much larger synthetic set of diagnostic values. The focus here is hurricane landfall, and we use landfall rates to evaluate the model. For each simulation, landfalls are computed in $100-\mathrm{km}$ segments along the Atlantic-Caribbean coast from the Yucatan Peninsula through Maine (Fig. 9). For each 1950-2008 synthetic set the landfalls are used to construct returnperiod curves, which are the average times between successive landfalls above a $V_{\max }$ threshold as a function of the threshold. Because of the stochastic nature of the simulations, a return-period curve is a statistic, and no two return-period curves are identical. These synthetic curves can be compared to the return-period curves computed directly from historic landfalls over the same period. The model is unbiased if the historic returnperiod curve falls within the inner $95 \%$ of the simulated curves. Figure 10 shows these return-period curves, with one panel for each of the 10 regions as labeled. In most regions at most intensity thresholds, the model is indeed unbiased by this standard. There is some evidence for a low bias at low $V_{\max }$ thresholds in the northern Gulf Coast.

Figure 11 shows the landfall rates (counts $\mathrm{yr}^{-1}$ ) along the full coast, Yucatan to the U.S. Northeast, in each intensity category separately. Shown are the model means across the 5000-member 1950-2008 simulation set, the $95 \%$ band about this mean, and the rates computed directly from HURDAT. For all categories but one, the HURDAT rate falls well inside the uncertainty range about the model mean, indicating the model landfall over the full coast is not biased for these intensities. For cat-1 landfalls, the HURDAT rate sits 
(a)

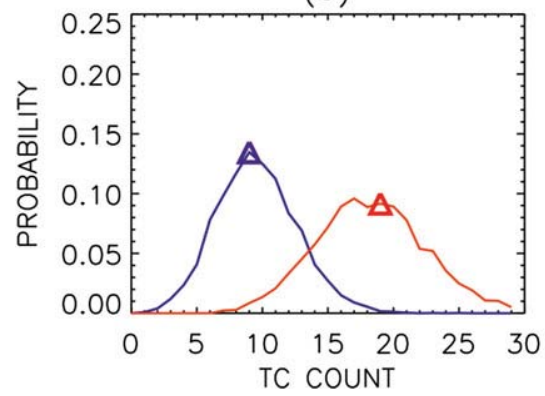

(b)

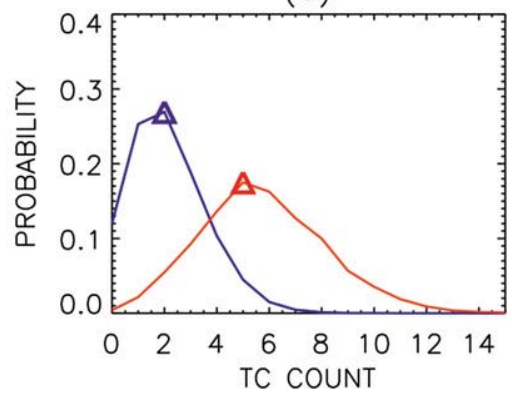

FIG. 12. Probability forecasts of NA TC counts in 2009 (blue curve) and 2010 (red curve) for (a) all named TCs and (b) major (cat-3+) hurricanes. The symbols indicate the actual numbers that occurred in 2009 (blue) and 2010 (red).

right at the top of the range, indicating a marginal low model bias for this category.

We further evaluate the model with an out-of-sample test of its forecast ability. The model has been constructed using HURDAT 1950-2008, but the 2-yr period $2009 / 10$ has also been included in the simulations. We compare these simulations to $2009 / 10$ observations. In other words, given observed 2009 and 2010 values for the independent variables SST and SOI, we test the model's probabilistic forecast of these years, which were not used in its construction. (A full forecast would require predictions for SST and SOI, as well; here, just the TC model is being tested.) The year 2009 was moderately unfavorable for NA TCs, with an SST anomaly of -0.4 and an SOI anomaly of 0.9 (El Niño), while 2010 was extremely favorable, with an SST anomaly of 3.1 and an SOI anomaly of -1.9 (La Niña).

Figure 12 shows the 2009 and 2010 distributions over the 5000-member simulation ensemble of NA-wide count of (i) all named TCs and (ii) all TCs that somewhere achieve major hurricane status (cat-3+). Although there is overlap between the 2009/10 distributions, there is a clear shift to more TCs in 2010. The ensemble-mean total TC counts are 9.6 (2009) and 18.3 (2010), and the ensemble-mean major hurricane counts are 2.1 (2009) and 5.8 (2010). The numbers that occurred in these years, shown as symbols in the figure, are 9 (2009) and 19 (2010) for total TCs and 2 (2009) and 5 (2010) for major hurricanes. These values are right at the peaks of the forecast probabilities, indicating good model forecasts.

Figure 13 shows the 2009/10 distributions over the 5000-member simulation ensemble of Yucatan-to-Maine landfalls of all named TCs and major hurricane status (cat-3+). The model does equally well for landfall forecast as for basinwide storm count. The mean forecast for all landfalls in 2009 is 3.8 and in 2010 is 7.2. The actual occurrence was 2 and 8 , both values near the peaks of their probabilistic forecasts. The mean forecast for major hurricane landfalls in 2009 is 0.4 and in 2010 is 1.1, while the actual occurrence was 0 and 1 (Hurricane Karl in 2010 on the Mexican Gulf Coast), again near the peak probabilistic forecast.

\section{Landfall and SST}

The primary goal of this study is to estimate the relationship between SST (or relSST) and landfall rates. For each of the five fixed values of SST anomaly $(-2,-1$, $0,+1$, and +2 ) we perform 10000 -yr simulations. The (a)

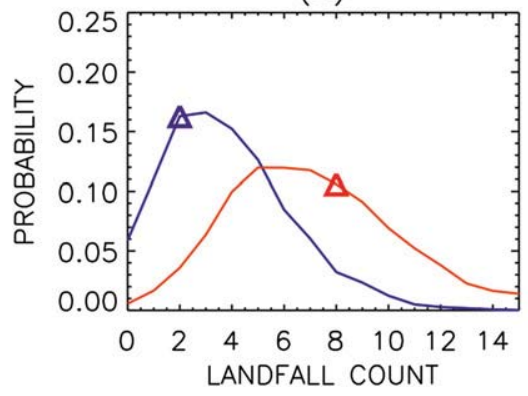

(b)

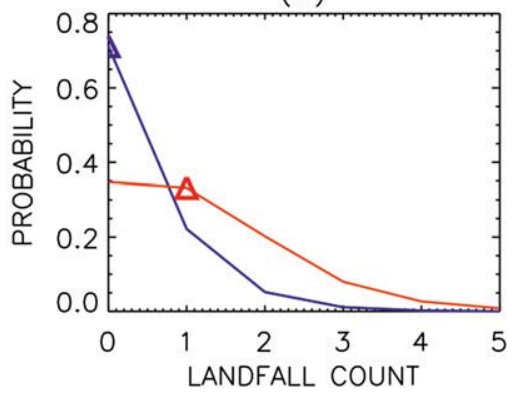

FIG. 13. As in Fig. 12, but for landfalling TCs along the full segmented coast shown in Fig. 9. 

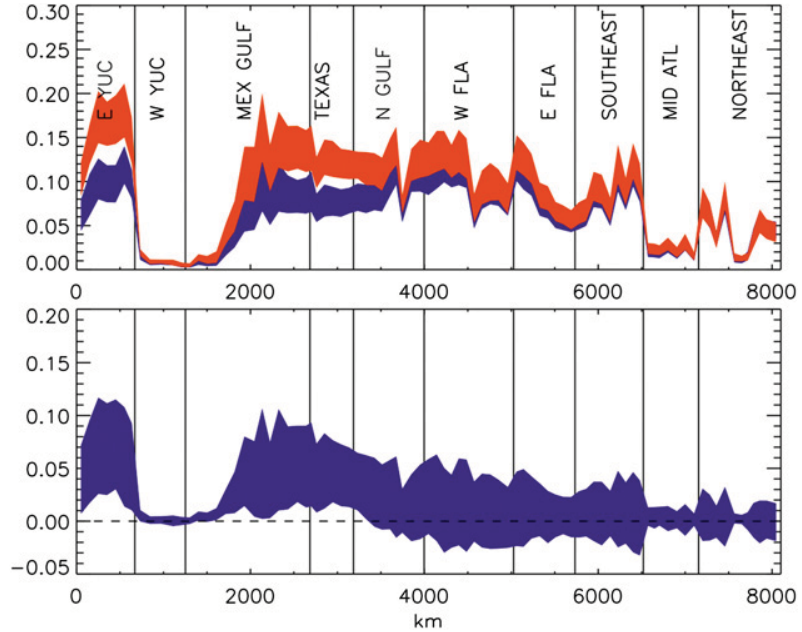

FIG. 14. (top) Probabilities along the U.S. and Mexican coasts (as labeled) of one or more TC landfalls of any intensity (cat- $0+$ ) per year per $100 \mathrm{~km}$ of coastline in +2 sigma SST years (red) and -2 sigma SST years (blue). (bottom) Difference in such probabilities hot minus cold. In each curve, the spread is a measure of significance, representing the inner $95 \%$ of results in a generalized jackknife test. In this figure, all of the model components genesis, propagation, and intensity are sensitive to SST.

long duration is necessary for the mean landfall rates at the $100-\mathrm{km}$ resolution to converge sufficiently and long return period to be estimated. We then compare mean landfall rates along the coast between the extreme +2 and -2 states.

It is crucial to estimate the uncertainty of the mean rates. A landfall rate difference between two SST states may be found, but is it significant? Uncertainty bounds about the means are determined with a generalized jackknife test. For each value of SST we perform 100 versions of the $10000-y r$ simulation. In each version, all model components are reconstructed on a random $80 \%$ subset of the data years 1950-2008 (i.e., a random 47 of the 59 years). This tests the sensitivity of the results to the finite nature of the underlying data on which the results are based. With this test we can say that the difference in mean landfall rates on some coastal region between the SST $=+2$ and SST $=-2$ states are significantly different (or not) if the inner $95 \%$ of the set of 100 differences excludes (or does not exclude) zero.

Figure 14 shows the probability of TC landfall at any intensity per year per $100-\mathrm{km}$ along the coast from the eastern Yucatan to Maine for warm (+2) and cold (-2) years. Also shown is the hot-cold difference. There is considerable geographic structure in the probabilities. In either hot or cold years, the eastern Yucatan and the northern Gulf Coasts have the greatest exposure, while the western Yucatan has almost no probability of a direct landfall. (Storms crossing the Yucatan east to west
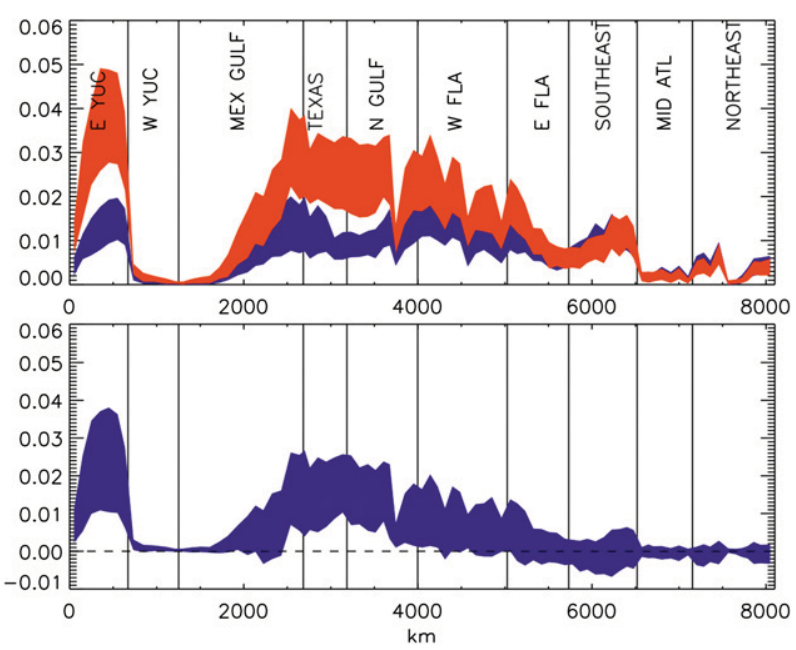

FIG. 15. As in Fig. 14, but for major hurricanes (cat-3+).

can do heavy damage on the west side of the peninsula. The diagnostic here counts storms as they actually cross ocean to land.) Figure 15 shows the same landfall probabilities but now for major hurricane landfalls (cat-3+). The geographic structure is similar to TC landfall at any intensity, though there is increased weight toward the Gulf and away from the U.S. East Coast and Northeast. Figure 16 shows the same major landfall probabilities but now with relSST as the independent variable. The results are very similar to SST, the biggest difference being western Florida, where the warm-year increase is marginally significant for SST but is insignificant for relSST.

With increased SST (or relSST), there is increased risk of landfall on the eastern Yucatan, on Texas, on the

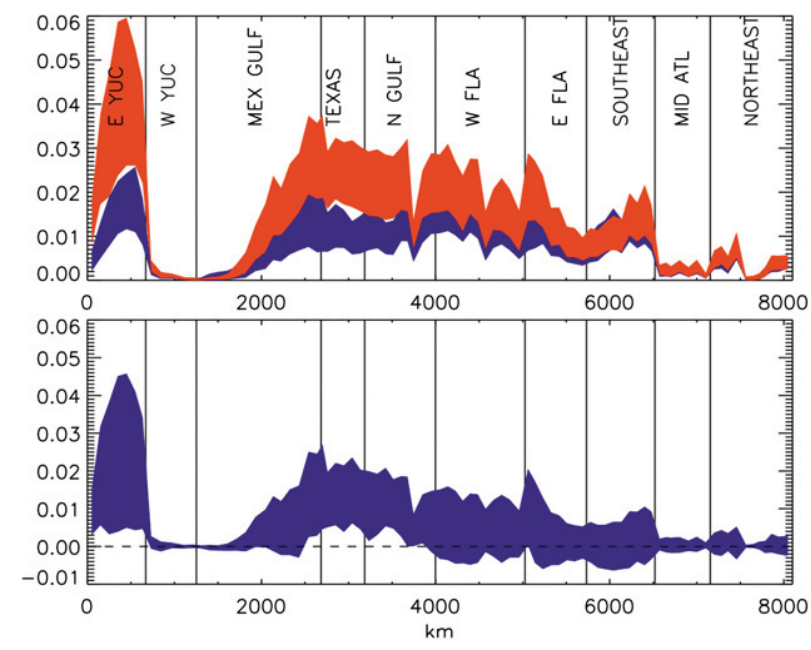

FIG. 16. As in Fig. 15, but the independent variable is relative SST (NA MDR JASO mean minus global northern subtropical JASO mean), with red +2 sigma and blue -2 sigma. 

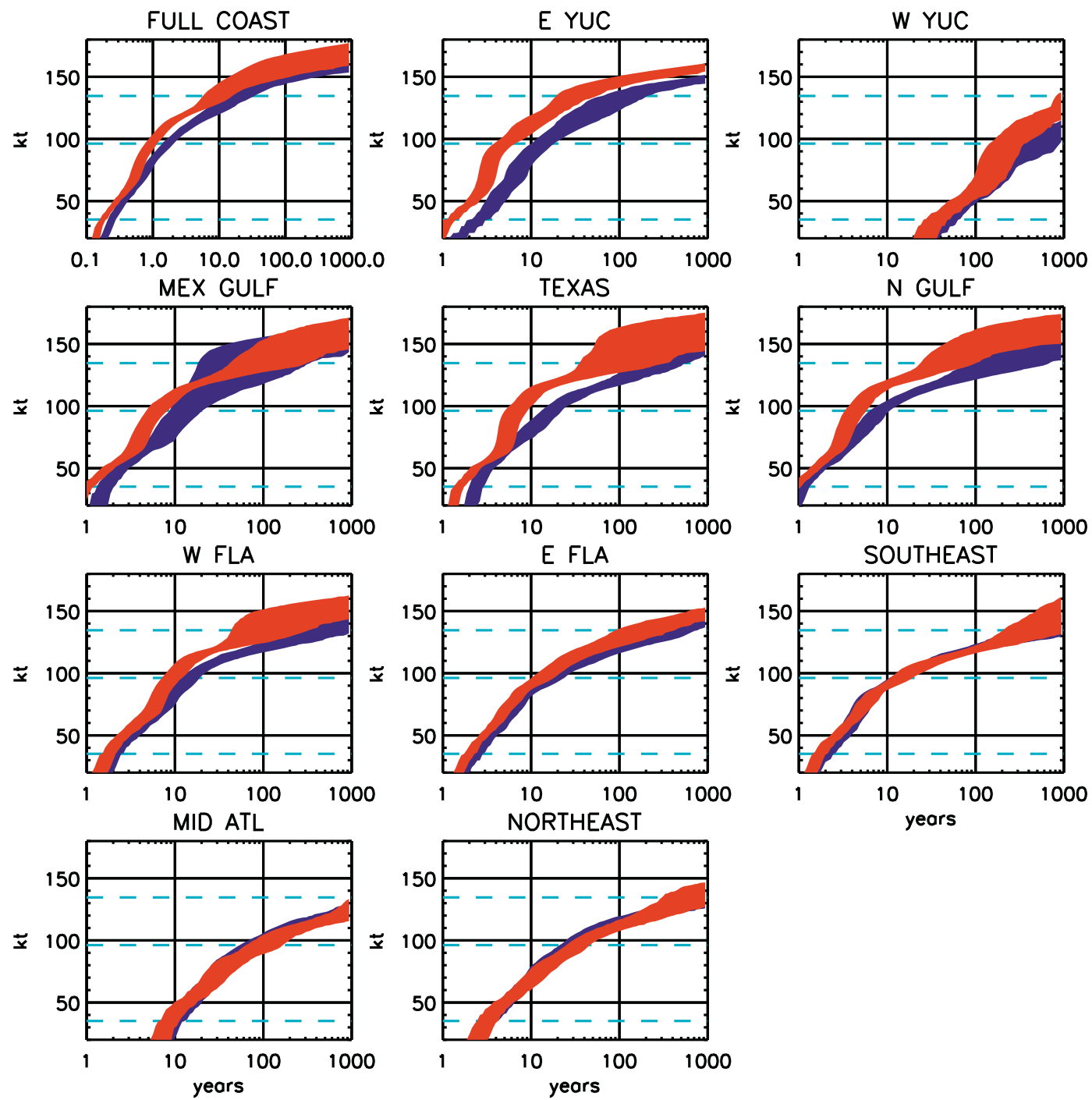

FIG. 17. Return-period curves for the fixed SST states +2 (red) and -2 (blue). The spread for each state indicates the $95 \%$ confidence bounds from the jackknife uncertainty test. The first panel includes the entire segmented coast of Fig. 9, while subsequent panels represent the smaller coastal regions of Fig. 9, as labeled. Note the different year range on the $x$ axis for the FULL COAST panel.

north Gulf Coast, and (for SST) marginally on the Florida Gulf Coast. The biggest increase is seen on the eastern Yucatan, where the probability of a cat-3+ landfall is 2 to 3 times larger for +2 SST than -2 SST. In Texas, the increase in landfall rate from -2 to +2 SST (or relSST) is roughly a factor 2. Interestingly, the U.S. East Coast from Florida through New England shows no significant sensitivity to SST or relSST. This is explored further below.

Figure 17 shows the SST $=+2$ and -2 landfall $-V_{\max }$ return-period curves for the full coast of Fig. 9 and for the individual regions. The spread for each SST state corresponds to the jackknife $95 \%$ confidence bounds. The eastern Yucatan coast has a reduction in return periods (increase in landfall frequency) that is significant at all intensities. That is, the red and blue regions are distinct at all intensities. Other Gulf Coast regions display a more varied SST sensitivity, typically showing a significant reduction in warm-year return periods for some intensities but not others. For example, Texas has significant return-period reduction for $V_{\max }$ less than $40 \mathrm{kt}\left(1 \mathrm{kt}=0.51444 \mathrm{~m} \mathrm{~s}^{-1}\right)$ and $V_{\max }$ between 80 and $120 \mathrm{kt}$. This complex signal is due to the tendency for warm-year return-period curves on the Gulf to become more vertical in the cat-2-3 intensity range, consistent with the observation that cat-2 and cat-3 landfall rates differ by relatively little (see cat- 2 and cat-3 landfall rates in Fig. 11). The U.S. East Coast, from Florida 

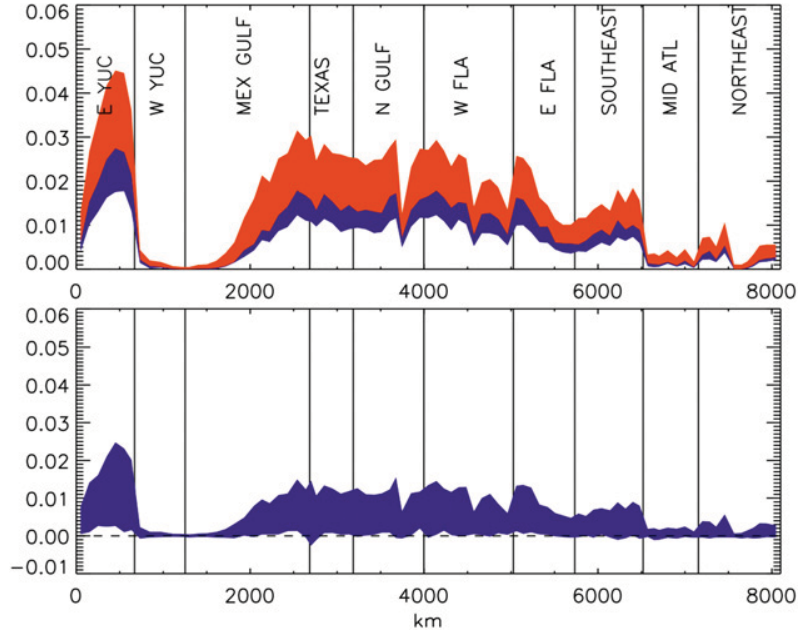

FIG. 18. As in Fig. 15, but only genesis is sensitive to SST.

through New England, shows no significant changes in landfall return periods at any intensity. Summed up for the full coast, there are significant reductions in return periods for all intensities up to about $120 \mathrm{kt}$, except for a small range around cat- 2 .

To help interpret the different sensitivities to SST along the coast, the entire analysis is repeated but now making only subsets of genesis, propagation, and intensity sensitive to SST. Figure 18 is similar to Fig. 15, except that only genesis is sensitive to SST. (That is, there is no SST regression in the propagation component, and the $V_{\max }$ sampling scheme now considers all years equally, independent of SST.) With only genesis sensitive to SST, there is marginally significant landfall increase everywhere along the Caribbean and Gulf Coasts through Florida. The increase on the U.S. East Coast is marginally insignificant. If TC genesis increased uniformly over the NA with SST (or relSST) then, without SST dependence in any other model component, we would expect the landfall rate change to be uniform along the coast. The modest geographic structure observed in the genesis-only landfall rate change must be because NA genesis does not change uniformly. Instead, the center of genesis shifts southeastward in warm years (Figs. 2 and 3).

When SST sensitivity is added to the track propagation (Fig. 19), the landfall profile looks more like that of the full model. The Yucatan shows no additional effect, the warm-year increase on the Mexican Gulf and Texas is exaggerated, and the rest of the Gulf and the U.S. East Coast no longer shows SST sensitivity. Figure 6 shows that in warm years TCs originating in the MDR do not reach as far west before recurving northeastward. This reduces the odds of a U.S. East Coast landfall, buffering the region from the overall increased TC count. Finally,
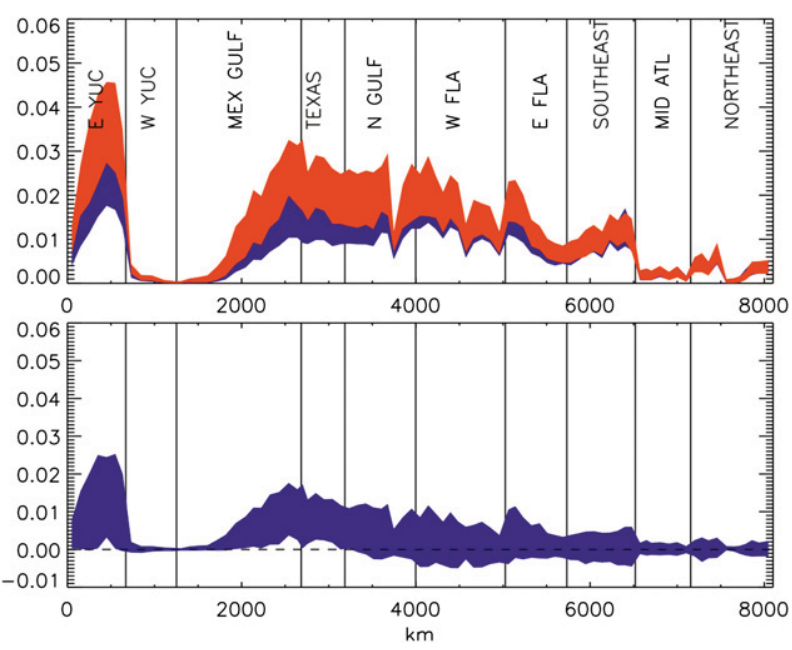

FIG. 19. As in Fig. 15, but only genesis and tracks (not $V_{\max }$ ) are sensitive to SST.

with $V_{\max }$ also sensitive to SST or relSST (Figs. 15 and 16), the Yucatan and Mexican Gulf sensitivities are exaggerated, Texas and the north Gulf have significant landfall increase with SST or relSST, and western Florida has significant increase with SST (but not relSST). The U.S. East Coast, however, remains insensitive to SST or relSST, mostly due to the buffering effect of track propagation changes, as well as the shift southeastward in the region of primary genesis.

To further illustrate the impact on landfall of factors beyond changes in basinwide counts, we plot in Fig. 20 the fraction of TCs of any intensity that make landfall per $100 \mathrm{~km}$ along the coast. That is, we divide the

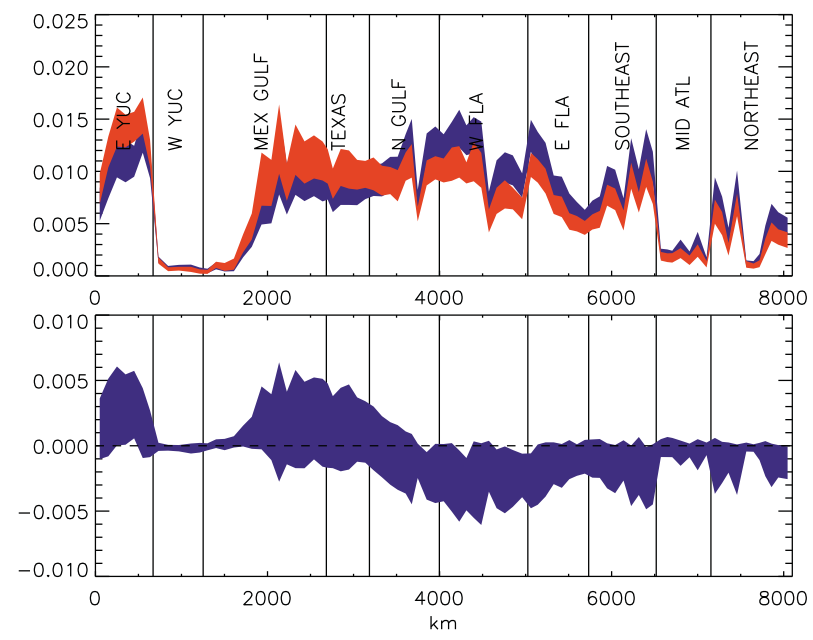

FIG. 20. (top) The fraction of all NA TCs that make landfall per $100 \mathrm{~km}$ of segmented coastline for hot (red) and cold (blue) SST. The spread indicates the inner $95 \%$ of the jackknife set. (bottom) The inner $95 \%$ of the hot-cold difference of the landfall fraction. 
(a)

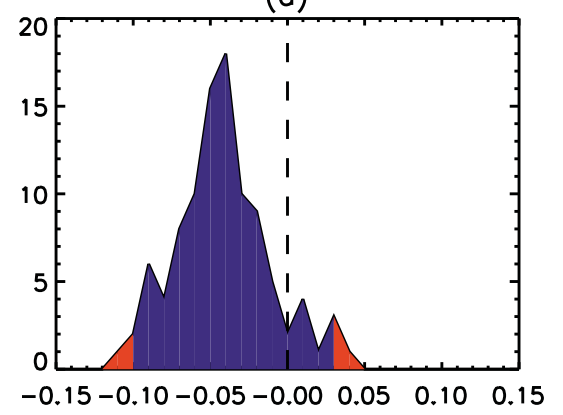

(b)

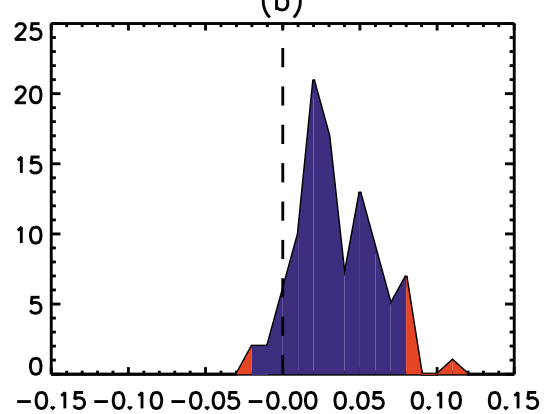

(c)

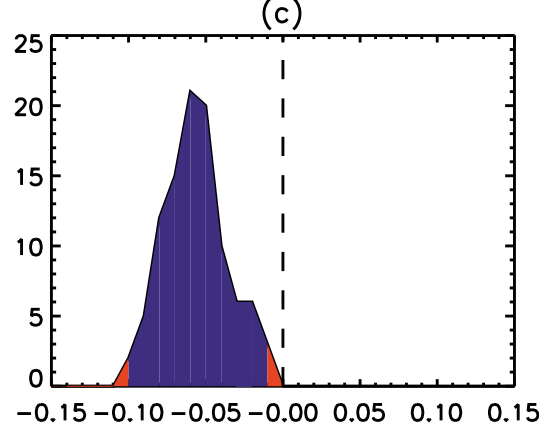

FIG. 21. Normalized histograms across the jackknife set of the hot-cold differences in landfall fraction of all NA TCs. Blue shows the inner $95 \%$ and red shows the upper and lower 2.5\% across the jackknife set. (a) The entire coastline, Yucatan to the U.S. Northeast. (b) The Yucatan and Mexican Gulf. (c) Florida through the Northeast.

landfall rates in the hot and cold SST simulations by the total hot and cold basinwide TC count. The landfall fraction depends on changes in genesis location and track with SST but not overall formation rate. From Florida through the Northeast there is a marginally significant decrease in landfall fraction with high SST compared to low. In contrast, from the Yucatan through the Mexican Gulf there is an increase warm over cold; the increase is significant on the eastern Yucatan coast but marginally insignificant on the Gulf Coast according to our jackknife uncertainty criterion.

Figure 21 summarizes these results in terms of the normalized histograms over the jackknife set of the difference of hot over cold landfall fraction, now summed over (i) the entire coast, (ii) the Yucatan through Mexican Gulf, and (iii) Florida through the U.S. Northeast. For the entire coast, there appears to be a decrease in landfall fraction with high SST, but the difference is not significant. The apparent increased landfall fraction on the Yucatan and Mexican Gulf is also not significant. The decrease on the region Florida through the Northeast is significant. In summary, we find no significant change with SST in the fraction of NA TCs making landfall anywhere from the Yucatan to the U.S. Northeast, in agreement with Villarini et al. (2012). However, the combined effects of shifts in genesis site and track changes hint at a dipole landfall effect, with a marginally insignificant increase in warm years on the Yucatan and western Gulf Coast, and a marginally significant decrease on the U.S. East Coast.

These results on the geographic distribution of landfall rate changes with SST are at least qualitatively in agreement with the work of Kossin et al. (2010). These authors decomposed NA TC tracks into four clusters and regressed the annual formation rate of each cluster on the AMM, which has a strong signature in NA SST. The cluster of TCs originating in the MDR and propagating westward to make western Gulf and Yucatan landfall is particularly sensitive to AMM. Its formation rate increases with increasingly positive AMM (and hence NA SST), leading to the enhanced western Gulf landfall rates we see in our analysis.

\section{Conclusions and discussion}

We have employed a statistical-stochastic model of TCs in the North Atlantic to estimate the sensitivity of North American TC landfall to SST and relative SST (relSST). We find statistically significant increases in annual major hurricane (cat-3+) landfall probability in warm years compared to cold for the eastern Yucatan, the Mexican Gulf Coast, Texas, and the north Gulf Coast. In addition, there is a significant increase on western Florida for SST, though not for relSST. Interestingly, there is no significant landfall change on the U.S. Atlantic coast, despite the overall increased annual TC count. Selective removal of the SST dependence from key model components reveals that the U.S. East Coast is buffered from the increased TC count by a shift with SST (and relSST) in track propagation from TCs originating in the MDR. In warm years, these tracks recurve to the northeast on average at a point farther east than in cold years, thereby reducing their odds of making East Coast landfall. Also contributing to this East Coast buffering is southeastward in the center of NA TC genesis and a consequent reduction in the landfall odds.

A purely statistical study such as this can make no statement about the physical mechanism of the SSTlandfall relationship. Kossin et al. (2010) show statistically that the SST patterns favorable for MDR TCs are high SST in the NA and low SST in the subtropical Pacific. Motivated by this and other studies we also tested relSST, in place of SST, as the independent predictor variable. The landfall results change little. Over the past 60 years, apparently, the climate state when SST is high, 
either absolutely or relatively, is associated with NA TC changes that cause significant landfall increases on parts of the North American coast. SST and/or relSST may be acting as a proxy for other TC-influencing variables associated with natural climate modes. For example, Kossin and Vimont (2007) discuss the Atlantic meridional mode (AMM) and its impact on the Atlantic warm pool and wind shear. In the AMM's positive phase, the North Atlantic is warm, providing a link to our SST and relSST predictors. The eastward expansion of the warm pool shifts the center of genesis eastward, which affects landfall rates, as TCs originating in the eastern MDR have lower odds of making North American landfall. Wang et al. (2011) have also argued that, via standing wave influence on the subtropical high-pressure system, the expanded warm pool influences TC propagation in precisely the way seen here: less westward penetration before northeastward recurvature, which reduces landfall odds.

Acknowledgments. We are grateful to Dr. Thomas Jagger for discussion concerning extreme value theory. We also thank three anonymous reviewers for helpful comments on the manuscript. This work was supported by a NASA National Climate Assessment Award and NOAA Grant NA11OAR4310093.

\section{REFERENCES}

Bender, M. A., T. R. Knudson, R. T. Tuleya, J. J. Sirutis, G. A. Vecchi, S. T. Garner, and I. M. Held, 2010: Modeled impact of anthropogenic warming on the frequency of intense Atlantic hurricanes. Science, 22, 454-458.

Camargo, S. J., 2013: Global and regional aspects of tropical cyclone activity in the CMIP5 models. J. Climate, in press.

_- K. Emanuel, and A. H. Sobel, 2007: Use of genesis potential index to diagnose ENSO effects upon tropical cyclone genesis. J. Climate, 20, 4819-4834.

Casson, E., and S. Coles, 2000: Simulation and extremal analysis of hurricane events. Appl. Stat., 49, 227-245.

Colbert, A. J., and B. J. Soden, 2012: Climatological variations in North Atlantic tropical cyclone tracks. J. Climate, 25, 657 673.

Elsner, J. B., and T. H. Jagger, 2008: United States and Caribbean tropical cyclone activity related to the solar cycle. Geophys. Res. Lett., 35, L18705, doi:10.1029/2008GL034431.

—, R. J. Murnane, and T. H. Jagger, 2006: Forecasting U.S. hurricanes 6 months in advance. Geophys. Res. Lett., 33, L10704, doi:10.1029/2006GL025693.

_ J. P. Kossin, and T. H. Jagger, 2008: The increasing intensity of the strongest tropical cyclones. Nature, 455, 92-95.

Emanuel, K., 2000: A statistical analysis of tropical cyclone intensity. Mon. Wea. Rev., 128, 1139-1152.

- 2005: Increasing destructiveness of tropical cyclones over the past 30 years. Nature, 436, 686-688.

, and T. Jagger, 2010: On estimating hurricane return periods. J. Appl. Meteor. Climatol., 49, 837-844.
—, S. Ravela, E. Vivant, and C. Risi, 2006: A statistical deterministic approach of hurricane risk assessment. Bull. Amer. Meteor. Soc., 87, 299-314.

_, R. Sundararajan, and J. Williams, 2008: Hurricanes and global warming: Results from downscaling IPCC AR4 simulations. Bull. Amer. Meteor. Soc., 89, 347-367.

Hall, T. M., and S. Jewson, 2007: Statistical modeling of North Atlantic tropical cyclone tracks. Tellus, 59A, 486-498.

$\longrightarrow$, and _ 2008: Comparison of local and basinwide methods for risk assessment of tropical cyclone landfall. J. Appl. Meteor. Climatol., 47, 361-367.

Holland, G. J., 1997: The maximum potential intensity of tropical cyclones. J. Atmos. Sci., 54, 2519-2541.

James, M. K., and L. B. Mason, 2005: Synthetic tropical cyclone database. J. Waterw. Port Coastal Ocean Eng., 131, 181192.

Javinen, B. R., J. Neumann, and M. A. Davis, 1984: A tropical cyclone data tape for the North Atlantic basin, 1886-1983: Contents, limitations, and uses. NOAA Tech. Memo. NWS NHC 22, 24 pp.

Kossin, J. P., and D. J. Vimont, 2007: A more general framework for understanding Atlantic hurricane variability and trends. Bull. Amer. Meteor. Soc., 88, 1767-1781.

- S. J. Camargo, and M. Sitkowski, 2010: Climate modulation of North Atlantic hurricane tracks. J. Climate, 23, 3057-3076.

Mendelsohn, R., K. Emanuel, S. Chonabayashi, and L. Bakkensen, 2012: The impact of climate change on global tropical cyclone damage. Nat. Climate Change, 2, 205-209.

Peduzzi, P., B. Chatenoux, H. Dao, A. De Bono, C. Herold, J. Kossin, F. Mouton, and O. Nordbeck, 2012: Global trends in tropical cyclone risk. Nat. Climate Change, 2, 289-294.

Ramsay, H. A., and A. H. Sobel, 2011: Effects of relative and absolute sea surface temperature on tropical cyclone potential intensity using a single-column model. J. Climate, 24, 183 193.

Rayner, N. A., D. E. Parker, E. B. Horton, C. K. Folland, L. V. Alexander, D. P. Rowell, E. C. Kent, and A. Kaplan, 2003: Global analysis of sea-surface temperature, sea ice, and night marine air temperature since the late nineteenth century. J. Geophys. Res., 108, 4407, doi:10.1029/2002JD002670.

Rumpf, J., H. Weindl, P. Hoppe, P. E. Raughe, and V. Schmidt, 2007: Stochastic modeling of tropical cyclone tracks. Math. Methods Oper. Res., 66, 475-490.

Santer, B. D., and Coauthors, 2006: Forced and unforced ocean temperature changes in Atlantic and Pacific tropical cyclogenesis regions. Proc. Natl. Acad. Sci. USA, 103, $13905-$ 13910.

Vecchi, G. A., and B. J. Soden, 2007: Effect of remote sea surface temperature change on tropical cyclone potential intensity. Nature, 450, 1066-1070.

_ - and T. R. Knudson, 2011: Estimating annual numbers of Atlantic hurricanes missing from the HURDAT data base (1878-1965) using ship track density. J. Climate, 24, 17361746.

- K. L. Swanson, and B. J. Soden, 2008: Whither hurricane activity? Science, 322, 687-689.

Vickery, P. J., J. Lin, P. S. Skerlj, L. A. Twisdale, and K. Huang, 2006: HAZUS-MH hurricane model methodology. I: Hurricane hazard, terrain, and wind load modeling. Nat. Hazards Rev., 7, 82-93.

Villarini, G., and G. A. Vecchi, 2012: Twenty-first-century projections of North Atlantic tropical storms from CMIP5 models. Nat. Climate Change, 2, 604-607. 
$\longrightarrow$, and -2013 : Projected increases in North Atlantic tropical cyclone intensity from CMIP5 models. J. Climate, 26, 32313240 .

- — - and J. A. Smith, 2010: Modeling the dependence of tropical storm counts in the North Atlantic basin on climate indices. Mon. Wea. Rev., 138, 2681-2705.

,,-- T. R. Knutson, and J. A. Smith, 2011: Is the recorded increase in short-duration North Atlantic tropical storms spurious? J. Geophys. Res., 116, D10114, doi:10.1029/2010JD015493.
- - - and J. A. Smith, 2012: U.S. landfalling and North Atlantic hurricanes: Statistical modeling of their frequencies and ratios. Mon. Wea. Rev., 140, 44-65.

Wang, C., L. Hailong, S.-K. Lee, and R. Atlas, 2011: Impact of the Atlantic warm pool on United States landfalling hurricanes. Geophys. Res. Lett., 38, L19702, doi:10.1029/2011GL049265.

Yonekura, E., and T. M. Hall, 2011: A statistical model of tropical cyclone tracks in the western North Pacific with ENSOdependent cyclogenesis. J. Appl. Meteor. Climatol., 50, 1725-1739. 